\title{
Simulation and theory for two-dimensional beam-plasma instability
}

\author{
Sumin Yi, ${ }^{1}$ Tongnyeol Rhee, ${ }^{1,2}$ Chang-Mo Ryu, ${ }^{2}$ and Peter H. Yoon ${ }^{3, a)}$ \\ ${ }^{1}$ National Fusion Research Institute, Eoeun-dong, Yuseong-gu, Daejeon 305-333, Republic of Korea \\ ${ }_{3}^{2}$ Department of Physics, Pohang University of Science and Technology, Pohang 790-751, Republic of Korea \\ ${ }^{3}$ Institute for Physical Science and Technology, University of Maryland, College Park, \\ Maryland 20742, USA
}

(Received 12 August 2010; accepted 1 December 2010; published online 29 December 2010)

\begin{abstract}
A comparative study of the dynamics of the electron beam-plasma system in two spatial dimensions is carried out by means of particle-in-cell (PIC) simulation and quasilinear theory. In the literature, the beam-plasma instability is usually studied with one-dimensional assumption. Among the few works that include higher-dimensional effects are two- and three-dimensional quasilinear theory and two-dimensional PIC simulations. However, no efforts were made to compare the theory and simulation side by side. The present paper carries out a detailed comparative study of two-dimensional simulation and quasilinear theory. It is found that the quasilinear theory quite adequately accounts for most important features associated with the simulation result. For instance, the particle diffusion time scale, the maximum wave intensity, dynamical development of the electron distribution function, and the change in the wave spectrum all agree quantitatively. However, certain nonlinear effects such as the Langmuir condensation phenomenon are not reproduced by the quasilinear theory. Nevertheless, the present paper verifies that the simple quasilinear theory is quite effective for the study of beam-plasma instability for the present choice of parameters. (C) 2010 American Institute of Physics. [doi:10.1063/1.3529359]
\end{abstract}

\section{INTRODUCTION}

The electron beam-plasma (or bump-on-tail) instability is a fundamental problem with a wide range of applications. For instance, it is at the core of solar type II and type III radio bursts. The beam-plasma interaction also characterizes the laboratory discharge experiments. The simplest nonlinear theory that may be employed to investigate the dynamical evolution of the beam-plasma system is the well-known quasilinear theory. ${ }^{1-5}$ Quasilinear theory is applicable to relatively early phase of nonlinear dynamics before mode-mode coupling takes place. Beyond quasilinear saturation phase the fully nonlinear dynamics can be further discussed by more sophisticated theories such as the weak turbulence theory. ${ }^{6-10}$

Quasilinear and weak turbulence theories are incoherent theories based upon phase averages. Consequently, these theories preclude coherent nonlinear processes such as the particle trapping at the outset. ${ }^{11-13}$ One-dimensional (1D) approximation for the beam-plasma interaction is often satisfactory when a strong magnetic field is present. In such a case, the electron beam streaming along the ambient magnetic field predominantly excites Langmuir waves propagating along $B$ field. However, for plasmas characterized by a weak magnetic field such as the solar wind near $1 \mathrm{AU}, 1 \mathrm{D}$ assumption for the wave excitation is not appropriate. Moreover, in the context of the solar type II and/or type III burst problem, the plasma emission rate depends on the angle between the wave vectors associated with the primary and backscattered Langmuir waves. ${ }^{14,15}$ As a matter of fact,

\footnotetext{
${ }^{a}$ Present addresses: School of Space Research, Kyung Hee University, Yongin, Gyeonggi 446-701, Korea and Massachusetts Technological Laboratory, Inc., 330 Pleasant Street, Belmont, MA 02478.
}

strictly parallel and antiparallel Langmuir wave pairs do not lead to the plasma emission. This makes the problem inherently two-dimensional (2D) or even three-dimensional (3D).

To investigate this problem with $1 \mathrm{D}$ approximation, $\mathrm{Li}$ et al. ${ }^{14}$ projected the 2D (or 3D with cylindrical symmetry) Langmuir wave spectrum onto one-dimension by assuming that the 2D Langmuir wave spectrum occupies an arc-shape domain in 2D wave number space. Their model is based upon numerical solutions of the Zakharov equations. ${ }^{15,16}$ Broadening of the Langmuir wave spectrum in the perpendicular direction to the beam is found by numerically integrating the Zakharov equations in two-dimension, ${ }^{16}$ which was attributed to three-wave decay processes.

In the literature, most analyses of the electron beamplasma interaction are performed under the simplifying 1D assumption. However, some theoretical and numerical works employ higher-dimensional effects. ${ }^{17-21}$ Bernstein and Engelmann ${ }^{18}$ examined asymptotic solution of 3D quasilinear equation. By employing the energy conservation principle, they found that 3D plasma is qualitatively different from a 1D plasma in that the Langmuir wave energy asymptotically decays away. Appert et al. ${ }^{19}$ numerically solved 2D quasilinear equation and confirmed Bernstein and Engelmann's conclusion.

The 2D particle-in-cell (PIC) simulations of the electron beam-plasma instability were carried out by Dum and Nishikawa ${ }^{20}$ and by Kasaba et al. ${ }^{21}$ Dum and Nishikawa ${ }^{20}$ presented time evolution of the Langmuir wave spectrum and showed that the wave propagation and diffusion in the perpendicular direction are quite significant. Kasaba et al. ${ }^{21}$ investigated the generation of electrostatic and electromagnetic second harmonic emissions. These PIC simulations also 
demonstrated that the Langmuir wave energy dissipation in 2D is much more pronounced than in 1D simulations.

In spite of all these studies, there still remains a necessity for further study of 2D Langmuir turbulence. For instance, theoretical works in Refs. 18 and 19 were largely concerned with asymptotic behavior. Simulation studies in Refs. 20 and 21 focused on either the second harmonic emissions or general Langmuir wave dynamics.

The purpose of this paper is to compare the predictions of the quasilinear theory and the PIC simulation result in 2D space. It should be noted, however, that in a recent series of works, 2D quasilinear particle equation as well as the weak turbulence wave kinetic equation for the Langmuir and ionacoustic turbulence were numerically solved. ${ }^{22-27}$ The wave kinetic equations considered in these references not only contain quasilinear (that is, linear wave-particle resonance) effects but also nonlinear wave-wave as well as nonlinear wave-particle interaction terms. In the present paper, only quasilinear theory is taken into account for the benchmark against the 2D PIC simulation. In this regard, the present study and Refs. 22-27 are mutually complementary. The PIC code includes not only quasilinear but fully nonlinear effects. In order to compare the PIC simulation results with quasilinear theory we shall limit our simulation to relatively short runs.

The organization of this paper is as follows: In Sec. II we present the quasilinear equations. Then in Sec. III we explain our PIC simulation scheme and setup. The simulation results are presented and interpreted in the light of quasilinear theory in Sec. IV. Subsequent two sections are devoted to the numerical analysis of quasilinear equations: The numerical algorithm is discussed in Sec. V, and Sec. VI presents the numerical solutions of the 2D quasilinear equations. Quantitative comparison to the PIC simulation results and differences thereof are also discussed. Finally, Sec. VII concludes the paper.

\section{EQUATIONS OF QUASILINEAR THEORY}

The equations of the quasilinear theory consist of the electron kinetic equation and the Langmuir wave kinetic equation. ${ }^{3-5,19,28,29}$ The particle kinetic equation for the electrons is given by

$$
\frac{\partial F_{e}}{\partial t}=\frac{\partial}{\partial v_{i}}\left(A_{i} F_{e}+D_{i j} \frac{\partial F_{e}}{\partial v_{j}}\right),
$$

where $A_{i}$ represents the drag term that arises from discreteparticle effects, and $D_{i j}$ is the velocity-space diffusion coefficient. They are defined by

$$
\begin{aligned}
& A_{i}=\frac{e^{2}}{4 \pi m_{e}} \int d \mathbf{k} \frac{k_{i}}{k^{2}} \omega_{\mathbf{k}}^{L} \delta\left(\omega_{\mathbf{k}}^{L}-\mathbf{k} \cdot \mathbf{v}\right), \\
& D_{i j}=\frac{\pi e^{2}}{m_{e}^{2}} \int d \mathbf{k} \frac{k_{i} k_{j}}{k^{2}} \delta\left(\omega_{\mathbf{k}}^{L}-\mathbf{k} \cdot \mathbf{v}\right) I_{\mathbf{k}}^{L} .
\end{aligned}
$$

Here, $F_{e}$ is the electron distribution function normalized to unity $\left[\int d \mathbf{v} F_{e}(\mathbf{v})=1\right], I_{\mathbf{k}}^{L}$ is the Langmuir spectral wave intensity, $\omega_{\mathbf{k}}^{L}$ and $\mathbf{k}$ are the Langmuir wave angular frequency and wave vector, respectively, $e$ is the unit electron charge, and $m_{e}$ is the electron mass. In the present discussion we ignore nonresonant interactions, as the distribution of nonresonant particles hardly change in the early phase of the beamplasma dynamics. ${ }^{4,5}$

Quasilinear Langmuir wave kinetic equation is given by

$$
\frac{\partial I_{\mathbf{k}}^{L}}{\partial t}=S_{\mathbf{k}}+2 \Gamma_{\mathbf{k}} I_{\mathbf{k}}^{L},
$$

where the spontaneous emission term, $S_{\mathbf{k}}$, and the linear growth/damping rate associated with the induced emission, $\Gamma_{\mathbf{k}}$, are given, respectively, by

$$
\begin{aligned}
& S_{\mathbf{k}}=\frac{n_{0} e^{2} \omega_{p e}^{2}}{k^{2}} \int d \mathbf{v} \delta\left(\omega_{\mathbf{k}}^{L}-\mathbf{k} \cdot \mathbf{v}\right) F_{e}, \\
& \Gamma_{\mathbf{k}}=\frac{\pi}{2} \omega_{\mathbf{k}}^{L} \frac{\omega_{p e}^{2}}{k^{2}} \int d \mathbf{v} \delta\left(\omega_{\mathbf{k}}^{L}-\mathbf{k} \cdot \mathbf{v}\right) \mathbf{k} \cdot \frac{\partial F_{e}}{\partial \mathbf{v}} .
\end{aligned}
$$

In the above, $\omega_{p e}$ is the electron plasma angular frequency defined by $\omega_{p e}=\left(4 \pi n_{0} e^{2} / m_{e}\right)^{1 / 2}$, where $n_{0}$ is the average plasma density.

\section{PIC SIMULATION SETUP}

The simulation is performed by using a $2 \mathrm{D}$ electrostatic PIC code with a periodic boundary condition. ${ }^{30}$ The simulation system is taken in the $x-y$ plane. The number of spatial grids is $512 \times 512$ and the total time steps are 10240 . The grid size, $\Delta x$, is equal to the electron Debye length $\lambda_{\text {De }}$, and the time step, $\Delta t$, is $0.1 \omega_{p e}^{-1}$. Here, $\lambda_{\mathrm{De}}$ is defined by $\lambda_{\mathrm{De}}$ $=\left(\epsilon_{0} T_{e} / n_{0} e^{2}\right)^{1 / 2}$ and $T_{e}$ is the electron temperature. The simulation is run till $t=1024 \omega_{p e}^{-1}$. The length and time are normalized by $x_{0}=\lambda_{\mathrm{De}}$ and $t_{0}=1 / \omega_{p e}$, respectively. The unit is chosen such that $\epsilon_{0}$ is unity.

The plasma consists of three species: the background electrons, ions, and the electron beam. The ratio of ion-toelectron mass, $m_{i} / m_{e}$, is set close to the real value, namely, 1600. The velocity distributions for the background electrons and ions are Maxwellian in all directions with thermal speed $v_{t s}=\left(2 T_{s} / m_{s}\right)^{1 / 2}(s=e, i$ denote the background electrons and ions, respectively). The ion temperature is $T_{i}=T_{e} / 10$. The beam electrons drift along $x$ axis with velocity $v_{b}=5 v_{t e}$. To reduce the numerical instability by the beam charge fluctuation, we used fractional charges and masses for the beam particles, yet keeping $q / m$ to be the same as that of the electron. Splitting beam electrons to fractional ones enhances the statistical accuracy. The number of beam electrons initially allocated is $10 \%$ of $n_{0}$, but because $1 / 5$ of the mass and charge are assigned for the beam electrons, the total beam charge density in fact becomes $2 \%$. In the simulation, the additional charge introduced by the beam violates the charge neutrality and thus the extra charge is artificially removed.

The initial velocity distribution for the electron beam is a drifting Maxwellian with the beam thermal speed equal to the background thermal speed, $v_{t b}=\left(2 T_{b} / m_{b}\right)^{1 / 2}=v_{t e}$. In our simulation, 1080 background electrons, 108 beam electrons, 
and 1080 ions are used in each cell. Initially, background electrons, the beam, and the ions are uniformly distributed in space.

In order to identify the Langmuir wave, we take the Fourier transformation of the electric field in both space and time and obtain the power spectrum associated with longitudinal electric field component by computing

$$
\left|E_{s}\right|^{2}=\frac{[\mathbf{k} \cdot \mathbf{E}(\mathbf{k}, \omega)]^{2}}{k^{2}} .
$$

Fourier transformation in time is then taken for the normalized time interval ranging from, $\tau=0-102, \tau=102-204$, and $\tau=614-716$, where $\tau$ is the normalized time defined by $\tau$ $=t \omega_{p e}$. The resolution in angular frequency is $0.06 \omega_{p e}$.

Theoretical Langmuir wave intensity is defined by

$$
\left|E_{s}(\mathbf{k}, \omega)\right|^{2}=I_{\mathbf{k}}^{L} \delta\left(\omega-\omega_{\mathbf{k}}^{L}\right) .
$$

However, the above definition ignores the contribution from fluctuations other than the plasma normal modes. The electric field intensity in the simulation contains contributions from both the plasma normal modes, satisfying $\epsilon(\mathbf{k}, \omega)=0$, and thermal fluctuations characterized by $\epsilon(\mathbf{k}, \omega) \neq 0$. Since it is not possible to single out only the normal mode contribution in the simulation, we define the Langmuir wave intensity as follows

$$
\iota(\mathbf{k})=C \int_{\omega_{\min }}^{\omega_{\max }} d \omega\left|E_{s}(\mathbf{k}, \omega)\right|^{2} .
$$

The integral range is limited to the vicinity of the plasma frequency, $\omega_{\min } \approx 0.8 \omega_{p e}$ and $\omega_{\max } \approx 1.2 \omega_{p e}$. This ensures that the contribution comes mainly from the plasma normal mode, i.e., the Langmuir wave. The coefficient, $C$, is determined by matching the initial thermal fluctuation levels for Langmuir waves obtained from the PIC simulation and theory. The electron distribution function in 2D velocity space, $F_{e}\left(v_{x}, v_{y}\right)$, is obtained by dividing the number of electrons in $2 \mathrm{D}$ velocity cell by the total number of electrons.

\section{SIMULATION RESULT}

Figure 1 plots the electron distribution function, $F_{e}\left(v_{x}, v_{y}\right)$, in $2 \mathrm{D}$ velocity space (left-hand panels) and the Langmuir wave intensity, $\iota\left(q_{x}, q_{y}\right)$, in $2 \mathrm{D}$ wave vector space (right-hand panels), at several times. Here, $q_{j}=k_{j} v_{t e} / \omega_{p e}$ $=\sqrt{2} k_{j} \lambda_{\mathrm{De}}$ corresponds to the normalized wave number. The temporal variable is normalized as $\tau=t \omega_{p e}$. 2D wave spectrum is shown on the right-hand panels from top to bottom in Fig. 1, for the time intervals ranging from $\tau=0-102, \tau$ $=102-204$, and $\tau=614-716$. The left panels show the change of electron distribution at $\tau=0,102$, and 614. For early times, $\tau<200$, the $2 \mathrm{D}$ evolution of the beam-plasma instability follows the well-known pattern largely described by 1D quasilinear scenario. That is, the beam distribution develops a velocity-space plateau by the time the system has evolved to $\tau \approx 200$ or so, and concomitantly, the Langmuir wave initially grows over the wave number space satisfying the resonance condition, $\omega \sim \mathbf{k} \cdot \mathbf{v}_{b}$, namely, $0.2<q_{x}<0.3$ and $\left|q_{y}\right|<0.3$. The Langmuir wave intensity reaches the saturation level around $\tau \approx 200$.
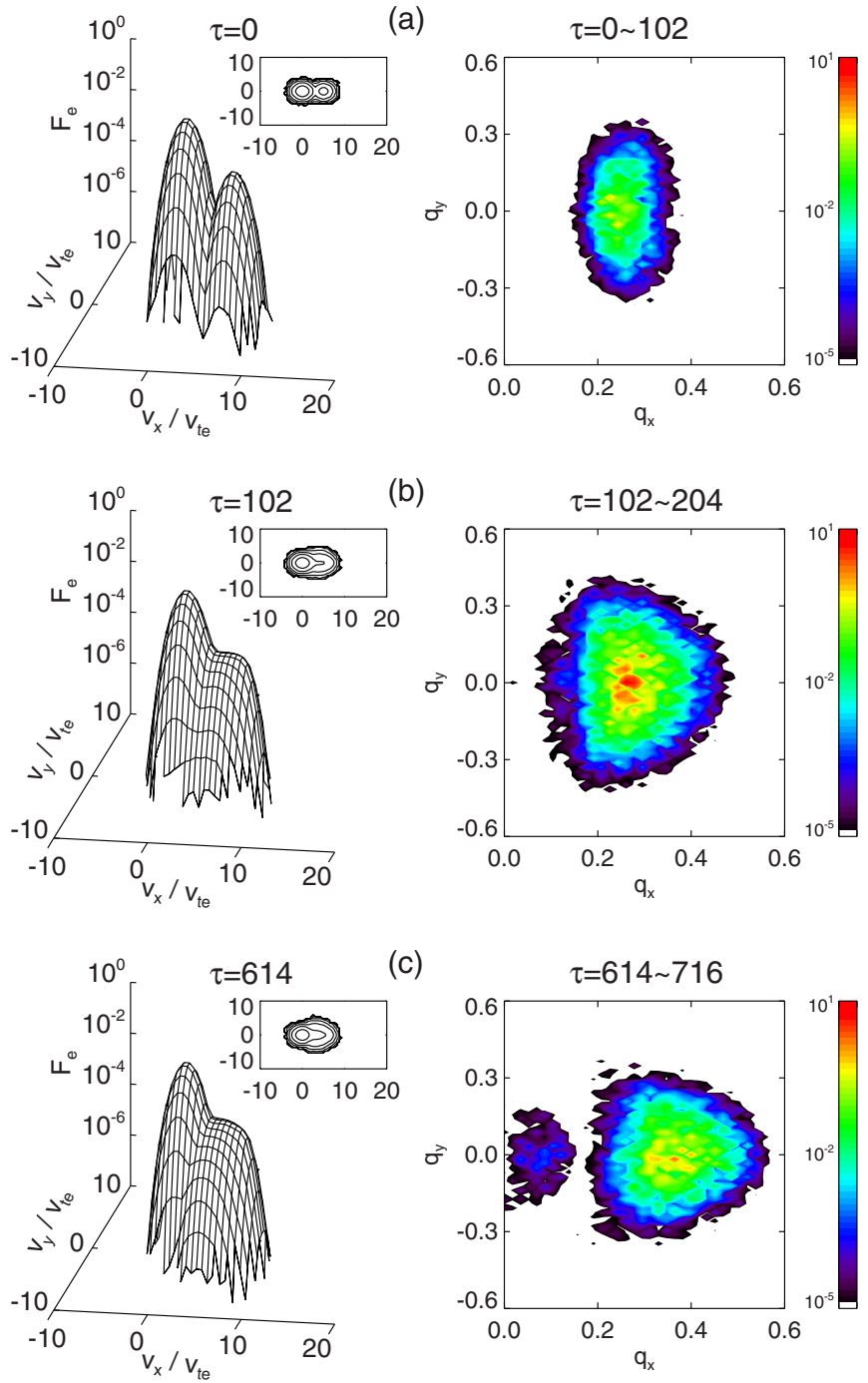

(c)

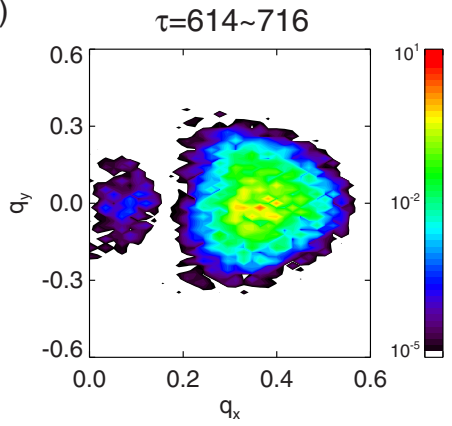

FIG. 1. (Color online) Electron distribution function, $F_{e}(\mathbf{v}, \tau)$, in 2D velocity space (left) and Langmuir wave intensity, $\iota(\mathbf{q}, \tau)$, in $2 \mathrm{D}$ wave number space (right) obtained from PIC simulation at (a) $\tau=0-102$, (b) $\tau$ $=102-204$, and (c) $\tau=614-716$.

For later times $(\tau \sim 102-204)$, it can be seen that the 2D spectrum of Langmuir waves begins to evolve into a D-shape pattern. This is consistent with Refs. 22 and 23 where it is reported that the nonlinear development of the beam-excited Langmuir waves should evolve into a ringlike pattern in 2D wave number space. Of course, the complete ring spectrum is not achieved in our run since the total simulation time is not sufficiently long. References 22 and 23 report that the quasiring spectrum fully develops on time scale of the order of $10^{3}-10^{4} \omega_{p e}^{-1}$.

The present result also justifies the arc-spectrum modeled in Refs. 15 and 16. These references model the arcspectrum on the basis of Zakharov theory but the present PIC simulation provides an independent verification of their model.

The excitation of long wavelength $\left(q_{x} \sim 0\right)$ Langmuir modes is observed for $\tau \sim 614-716$. The linear wave-particle interaction is not responsible for the excitation of these modes. These condensate modes seem to grow as a result of nonlinear processes. ${ }^{31-33}$ According to the $2 \mathrm{D}$ weak turbu- 

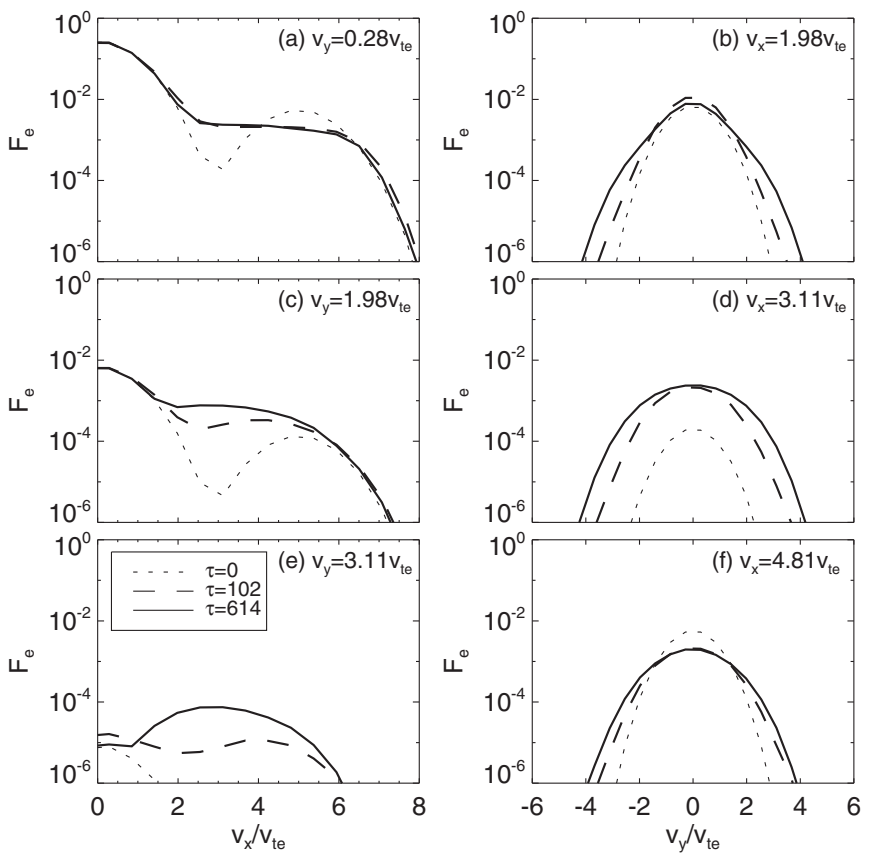

FIG. 2. Cross sections of the electron distribution function shown in Fig. 1 taken along $v_{x}$ for fixed $v_{y}^{\prime}$ (left panels) and along $v_{y}$ for fixed $v_{x}^{\prime} s$ (right panels). The left-hand panels show to cross sections taken at (a) $v_{y}$ $=0.28 v_{t e}$, (c) $v_{y}=1.98 v_{t e}$, and (e) $v_{y}=3.11 v_{t e}$. The right-hand panels shows perpendicular cross sections at (b) $v_{x}=1.98 v_{t e}$, (d) $v_{x}=3.11 v_{t e}$, and (f) $v_{x}$ $=4.81 v_{t e}$. For each panel we superposed the distributions at three different times, $\tau=0,102$, and 614 .

lence theory of Refs. 22 and 23, however, unlike 1D, the condensate mode does get excited, but the present simulation shows a condensate mode excitation. The exact reason for the discrepancy between 2D nonlinear theory and the present $2 \mathrm{D}$ simulation is not known at this time.

We also observe that the total Langmuir wave intensity decreases after it reaches the maximum value around the quasilinear plateau stage. The result is not shown, however. In the $1 \mathrm{D}$ case, the Langmuir wave saturates after the plateau formation in the electron distribution function, since the linear growth rate, $\Gamma_{k} \sim \partial F_{e} / \partial v=0$, becomes zero. In the 2D case, on the other hand, the Langmuir wave experiences further damping beyond the plateau formation. This is because the wave damping in the $2 \mathrm{D}$ case is determined not only by the parallel derivative, $\partial F_{e} / \partial v_{x}$, but also the perpendicular derivative of the electron distribution function, $\partial F_{e} / \partial v_{y}$ [see Eq. (4)]. Although the parallel derivative become zero after the plateau formation, the linear damping rate does not diminish owing to the contribution from the nonzero perpendicular derivative. This is consistent with the asymptotic analysis of 2D and 3D quasilinear theory in Refs. 18 and 19 who reported gradual decrease in the Langmuir wave intensity for multidimensional system.

According to Fig. 1, it can be seen that Langmuir waves that are initially in the nongrowing short-wavelength portion of the parallel wave number space $\left(0.35<q_{x}<0.5\right)$ begin to grow for later times $(\tau>102$ and beyond). It turns out that, coincident with the wave excitation, the electron distribution develops new positive gradients in small $v_{x}$ and finite $v_{y}$ range. The two phenomena, namely, the excitation of short- wavelength modes and the formation of the new population inversion, are related as we shall discuss more fully.

In Fig. 2, the cross sections of the 3D plots are presented to illustrate more detailed features of the evolution. In Fig. 2, we display the parallel (left panels) and perpendicular (right panels) electron distribution functions shown in Fig. 1. The parallel cross sections are taken at three different perpendicular velocities: (a) $v_{y}=0.28 v_{t e}$, (c) $v_{y}=1.98 v_{t e}$, and (e) $v_{y}$ $=3.11 v_{\text {te }}$, while the perpendicular cross sections are taken at parallel velocities given by (b) $v_{x}=1.98 v_{t e}$, (d) $v_{x}=3.11 v_{t e}$, and (f) $v_{x}=4.81 v_{t e}$. For each panel we superposed the distributions at three different times, $\tau=0,102$, and 614 .

Figure 2(a) shows that the parallel cut in the distribution taken at small $v_{y}$ resembles the $1 \mathrm{D}$ quasilinear picture in that the initial core-plus-beam distribution can be seen to evolve into a flattened plateau distribution at later times. However, the parallel cross sections taken at increasingly higher values of $v_{y}$, especially Fig. 2(e), show the formation of population inversion at late times, $\tau=614$. The new positive gradient that develops late in time occurs near the parallel velocity range $0<v_{x} / v_{t e}<2$. This newly developed positive gradients in small parallel velocities lead to the excitation of the Langmuir waves in relatively higher parallel wave numbers.

The parallel velocity satisfying the $2 \mathrm{D}$ wave-particle resonance condition, $\omega_{\mathbf{k}}-k_{x} v_{x}-k_{y} v_{y}=0$, is given by

$$
\left(v_{x}\right)_{\mathrm{res}}=\frac{\omega_{\mathbf{k}}-k_{y} v_{y}}{k_{x}} .
$$

In $1 \mathrm{D}$ or for small $v_{y}$, the resonant parallel velocity, $\left(v_{x}\right)_{\text {res }}$ $=\omega_{\mathbf{k}} / k_{x} \approx v_{b}$, is restricted to the vicinity of the beam speed. However, in 2D electrons having small $v_{x}$ but finite $v_{y}$ can satisfy the wave-particle resonance condition.

The perpendicular cross sections of the distributions shown in Figs. 2(b), 2(d), and 2(f) show that both the bulk and beam electrons undergo slight perpendicular heating. The broadening of the electron distribution functions in both perpendicular and parallel direction was observed in previously 2D quasilinear analysis. ${ }^{19,22,23}$

We now discuss the details associated with the Langmuir wave spectrum by considering parallel and perpendicular cuts along $q_{x}$ and $q_{y}$, respectively. Plotted in Fig. 3 are parallel (left-hand panels) and perpendicular (right-hand panels) Langmuir wave spectral distributions. For the parallel cross sections, fixed perpendicular wave numbers (a) $q_{y}=0$, (c) $q_{y}=0.052$, and (e) $q_{y}=0.156$, are considered, and for the perpendicular cross sections, (b) $q_{x}=0.208$, (d) $q_{x}=0.295$, and (f) $q_{x}=0.399$, are chosen. Initially, Langmuir waves in the range $0.2<q_{x}<0.3$ for all $q_{y}$ begin to grow, as the left panels show, and reach maximum intensity at $\tau=100-200$. After the saturation, part of the Langmuir wave spectrum damps while other parts continue to grow. We associate this shift in the Langmuir wave spectrum with the formation of positive gradients at late times, as already been discussed in Fig. 2(e). Parallel wave number $q_{x}$ associated with the waves at later times move to higher value owing to damping for small $q_{x}$ and growth for high $q_{x}$. The shift and broadening of the Langmuir wave spectrum was also found in previous 2D quasilinear studies. ${ }^{19,22,23}$ 
Note the excitation of the long-wavelength modes in the range $q_{x}<0.2$ for time steps beyond $\tau \approx 200$. This is the Langmuir condensate formation, which is usually discussed in the framework of 1D weak turbulence theory. ${ }^{31-33}$ However, the recent 2D weak turbulence theory suggests that such a condensate formation might be prohibited in $2 \mathrm{D}^{22,23}$ Nonetheless, the present PIC simulation shows that the Langmuir condensation is permitted after all. As noted previously the exact reason for the discrepancy is not known at this time, but this calls for further theoretical investigation of this phenomenon in higher dimensions.

Consider the perpendicular cross sections for $q_{x}=0.208$ [Fig. 3(b)]. Note that the initially growing waves undergo significant damping over time. The wave spectrum for $q_{x}$ $=0.295$ [Fig. 3(d)] is an intermediate case, but for $q_{x}$ $=0.399[$ Fig. $3(\mathrm{f})]$, the waves actually increase in magnitude over time. Again, this is consistent with previous studies $^{19,22,23}$ that show broadening and shifting of the wave spectrum in 2D wave number space. As a matter of fact, Refs. 22 and 23 show that over even longer time than is presently simulated, the Langmuir wave spectrum should evolve into a quasiring structure in 2D wave number space. On the other hand, Refs. 14 and 15 modeled the 2D Langmuir wave with an arc-shape spectrum. The previous works are thus consistent with the present simulation.

\section{NUMERICAL ANALYSIS OF QUASILINEAR THEORY}

To solve the quasilinear equations numerically we normalize Eqs. (1)-(4) as follows

$$
\begin{aligned}
& w=\frac{\omega}{\omega_{p e}}, \quad \mathbf{q}=\frac{\mathbf{k} v_{t e}}{\omega_{p e}}, \quad \tau=\omega_{p e} t, \\
& \mathbf{u}=\frac{\mathbf{v}}{v_{t e}}, \quad f(\mathbf{u})=v_{t e}^{3} F_{e}(\mathbf{v}), \quad I(\mathbf{q})=\frac{(2 \pi)^{2} \hat{g} I_{\mathbf{k}}^{L}}{\left(m_{e} v_{t e}^{2}\right)},
\end{aligned}
$$

where $v_{t e}=\left(2 T_{e} / m_{e}\right)^{1 / 2}, T_{e}$ being the electron temperature. In the above, the quantity $\hat{g}$ is an effective plasma parameter

$$
\hat{g}=\frac{1}{n_{0} \lambda_{\mathrm{De}}^{3}} \frac{1}{2^{3 / 2}(4 \pi)^{2}} \text {. }
$$

According to the above definition, the total Langmuir wave intensity is given by $\int d \mathbf{q} I(\mathbf{q})=\int d \mathbf{k} I_{\mathbf{k}}^{L} /\left(8 n_{0} T_{e}\right)$. The normalized dispersion relation is given by

$$
w_{\mathbf{q}}^{L}=\left\{\begin{array}{cc}
1+3 q^{2} / 4, & q_{x} \geq 0 \\
-\left(1+3 q^{2} / 4\right), & q_{x}<0
\end{array} .\right.
$$

Normalized electron quasilinear diffusion equation, (1) and (2), in 2D velocity space is given by

$$
\begin{aligned}
& \frac{\partial f}{\partial \tau}=\frac{\partial}{\partial u_{i}}\left(\bar{A}_{i} f+\bar{D}_{i j} \frac{\partial f}{\partial u_{j}}\right), \quad(i, j=x, y), \\
& \left(\begin{array}{l}
\bar{A}_{x} \\
\bar{A}_{y}
\end{array}\right)=\frac{4 \hat{g}}{3} \int_{-\infty}^{\infty} \frac{d q_{y}}{\sqrt{\Lambda\left(q_{y}\right)}} \sum_{q_{x}=q_{r 1}, q_{r 2}} \frac{1+3\left(q_{x}^{2}+q_{y}^{2}\right) / 4}{q_{x}^{2}+q_{y}^{2}}\left(\begin{array}{l}
q_{x} \\
q_{y}
\end{array}\right),
\end{aligned}
$$
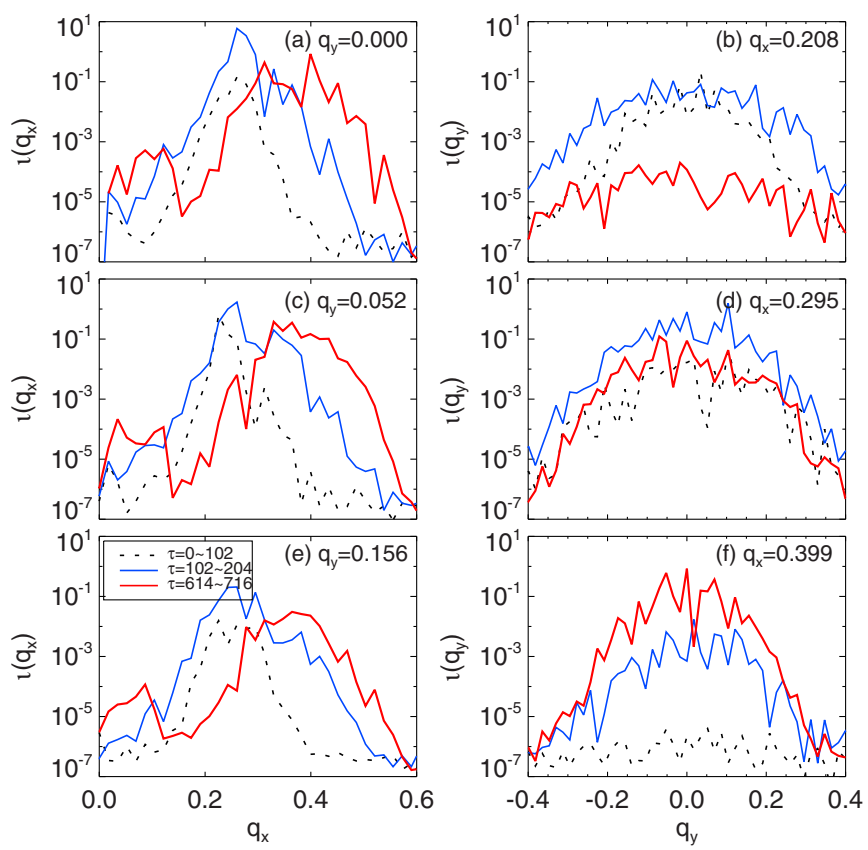

FIG. 3. (Color online) Parallel (left-hand panels) and perpendicular (righthand panels) cross sections of the 2D Langmuir wave intensity shown in Fig. 1.

$$
\left(\begin{array}{c}
\bar{D}_{x x} \\
\bar{D}_{x y} \\
\bar{D}_{y y}
\end{array}\right)=\frac{4}{3} \int_{-\infty}^{\infty} \frac{d q_{y}}{\sqrt{\Lambda\left(q_{y}\right)}} \sum_{q_{x}=q_{r 1}, q_{r 2}} \frac{I\left(q_{x}, q_{y}\right)}{q_{x}^{2}+q_{y}^{2}}\left(\begin{array}{c}
q_{x}^{2} \\
q_{x} q_{y} \\
q_{y}^{2}
\end{array}\right) .
$$

In the above, $\bar{D}_{x y}=\bar{D}_{y x}$, and $q_{x}=q_{r 1}, q_{r 2}$ are normalized resonant parallel wave numbers

$$
q_{r 1}=\frac{2}{3} u_{x}-\sqrt{\Lambda}, \text { and } q_{r 2}=\frac{2}{3} u_{x}+\sqrt{\Lambda}
$$

and the quantity $\Lambda$ is defined by

$$
\Lambda=\frac{4}{9} u_{x}^{2}-q_{y}^{2}+\frac{4}{3} u_{y} q_{y}-\frac{4}{3} .
$$

Normalized Langmuir wave kinetic equation is given by

$$
\begin{aligned}
& \frac{\partial I(\mathbf{q})}{\partial \tau}=s_{\mathbf{q}}+2 \gamma_{\mathbf{q}} I(\mathbf{q}), \\
& s_{\mathbf{q}}=\hat{g} \frac{\pi}{q^{2}} \int d \mathbf{u} \delta\left(w_{\mathbf{q}}^{L}-\mathbf{q} \cdot \mathbf{u}\right) f(\mathbf{u}), \\
& \gamma_{\mathbf{q}}=\frac{\pi}{2} \frac{w_{\mathbf{q}}^{L}}{q^{2}} \int d \mathbf{u} \delta\left(w_{\mathbf{q}}^{L}-\mathbf{q} \cdot \mathbf{u}\right) \mathbf{q} \cdot \frac{\partial f(\mathbf{u})}{\partial \mathbf{u}} .
\end{aligned}
$$

After evaluation of the velocity integral with the delta function, the spontaneous emission term and linear growth/ damping rate can be written as

$$
s_{\mathbf{q}}=\frac{\pi \hat{g}}{q^{2}}\left[\frac{1}{\left|q_{x}\right|} \int_{-\infty}^{\infty} d u_{y} f\left(\nu_{x}, u_{y}\right)+\frac{1}{\left|q_{y}\right|} \int_{-\infty}^{\infty} d u_{x} f\left(u_{x}, \nu_{y}\right)\right],
$$




$$
\begin{aligned}
\gamma_{\mathbf{q}}= & \frac{\pi}{2} \frac{w_{\mathbf{q}}^{L}}{q^{2}}\left\{\frac{q_{x}}{\left|q_{x}\right|} \int_{-\infty}^{\infty} d u_{y}\left[\frac{\partial f\left(u_{x}, u_{y}\right)}{\partial u_{x}}\right]_{u_{x}=\nu_{x}}\right. \\
& \left.+\frac{q_{y}}{\left|q_{y}\right|} \int_{-\infty}^{\infty} d u_{x}\left[\frac{\partial f\left(u_{x}, u_{y}\right)}{\partial u_{y}}\right]_{u_{y}=\nu_{y}}\right\},
\end{aligned}
$$

respectively. In the above equation $\nu_{x, y}$ are resonant parallel velocities given by

$$
\nu_{x}=\frac{w_{\mathbf{q}}^{L}-q_{y} u_{y}}{q_{x}}, \nu_{y}=\frac{w_{\mathbf{q}}^{L}-q_{x} u_{x}}{q_{y}} .
$$

Equations (10) and (11) are solved using the ADI method. ${ }^{34}$ We use $2 \mathrm{D}$ velocity and wave vector spaces corresponding to $-10<u_{x}<10$ and $-10<u_{y}<10$, and $1 \times 10^{-6}<q_{x}<0.8$ and $-0.8<q_{y}<0.8$, respectively. ${ }^{35}$

In the initial equilibrium, the Langmuir waves are stationary, $\partial I(\mathbf{q}) / \partial \tau=0$. The initial Langmuir wave spectrum is determined by balancing the spontaneous and induced emissions. ${ }^{36}$ Considering a thermal electron distribution, $f(\mathbf{u})=\pi^{-1} \exp \left(-u^{2}\right)$, the initial wave spectrum is computed from the wave kinetic Eq. (11)

$$
0=\int d \mathbf{u} \delta\left(w_{\mathbf{q}}^{L}-\mathbf{q} \cdot \mathbf{u}\right)\left[\hat{g}-2\left(w_{\mathbf{q}}^{L}\right)^{2} I(\mathbf{q})\right] \exp \left(-u^{2}\right)
$$

from which we obtain

$$
I(\mathbf{q}, 0)=\frac{\hat{g}}{2\left(w_{\mathbf{q}}^{L}\right)^{2}}=\frac{\hat{g}}{2+3 q^{2}} .
$$

If one ignores the spontaneous emission term, as is typical in many studies, then the initial spectrum becomes arbitrary. For instance, in Ref. 19 the initial wave spectrum was arbitrarily taken as a constant in the range of $10^{-2}-10^{-6}$ times the saturated wave intensity. In contrast, the initial wave spectrum is rigorously calculated in our study. In the PIC simulation the plasma parameter, $g=1 / n_{0} \lambda_{\text {De }}^{2}$, is approximately equal to $10^{-3}$ since we use 1080 background electrons and ions for each cell whose area is $\lambda_{\mathrm{De}}^{2}$. Consequently, we choose the similar value of $g$ in the numerical analysis of quasilinear theory.

The initial electron distribution function is a combination of a Maxwellian core distribution and a drifting Maxwellian beam distribution

$$
\begin{aligned}
f(\mathbf{u}, 0)= & \left(1-\frac{n_{b}}{n_{0}}\right) \frac{\exp \left[-\left(u_{x}^{2}+u_{y}^{2}\right)\right]}{\pi} \\
& +\frac{n_{b} / n_{0}}{\pi \alpha_{b}} \exp \left[-\frac{\left(u_{x}-u_{b}\right)^{2}+u_{y}^{2}}{\alpha_{b}^{2}}\right] .
\end{aligned}
$$

The beam-to-background electron number density is $n_{b} / n_{0}$ $=0.02$. The normalized beam drift speed and the beam thermal speed are chosen in accordance with the simulation setup, namely, $u_{b}=5.0$ and $\alpha_{b}=1.0$.

Figure 4 shows $f\left(u_{x}, u_{y}\right)$ and $I\left(q_{x}, q_{y}\right)$ computed on the basis of theoretical equations. The format is the same as Fig. 1. Major features found in the PIC simulation are reproduced, namely, the evolution of the particle distribution, and the formation of D-shaped wave spectrum, as well as the shifting and broadening of the spectrum. The fact that the
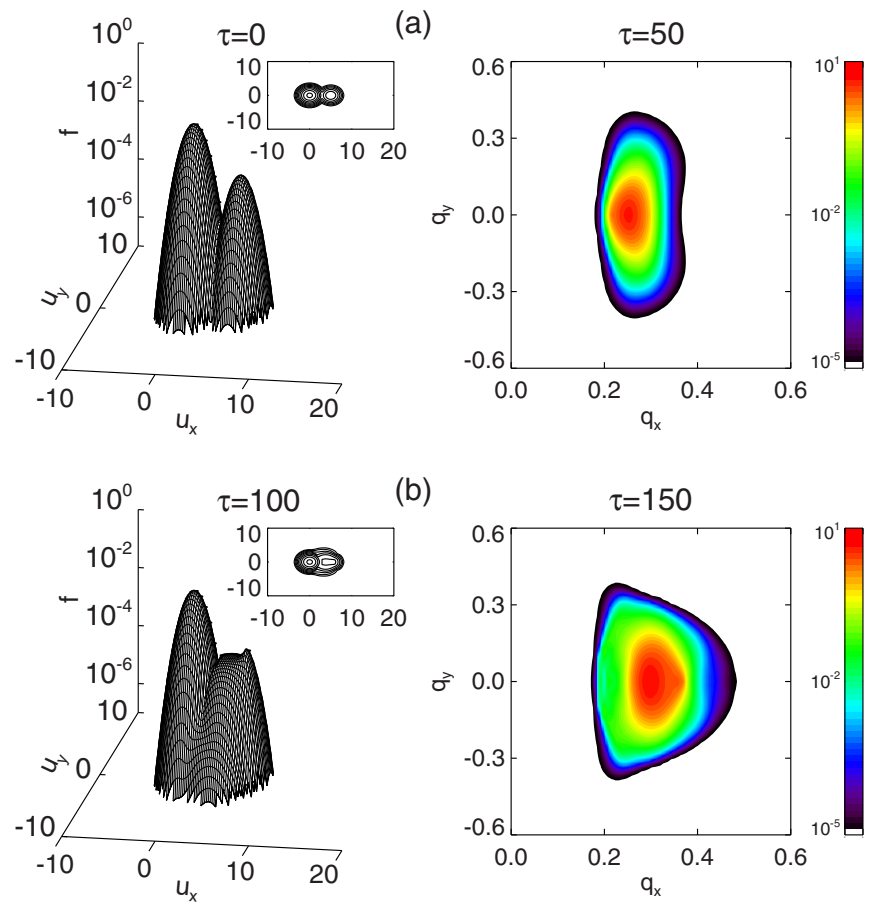

(b)
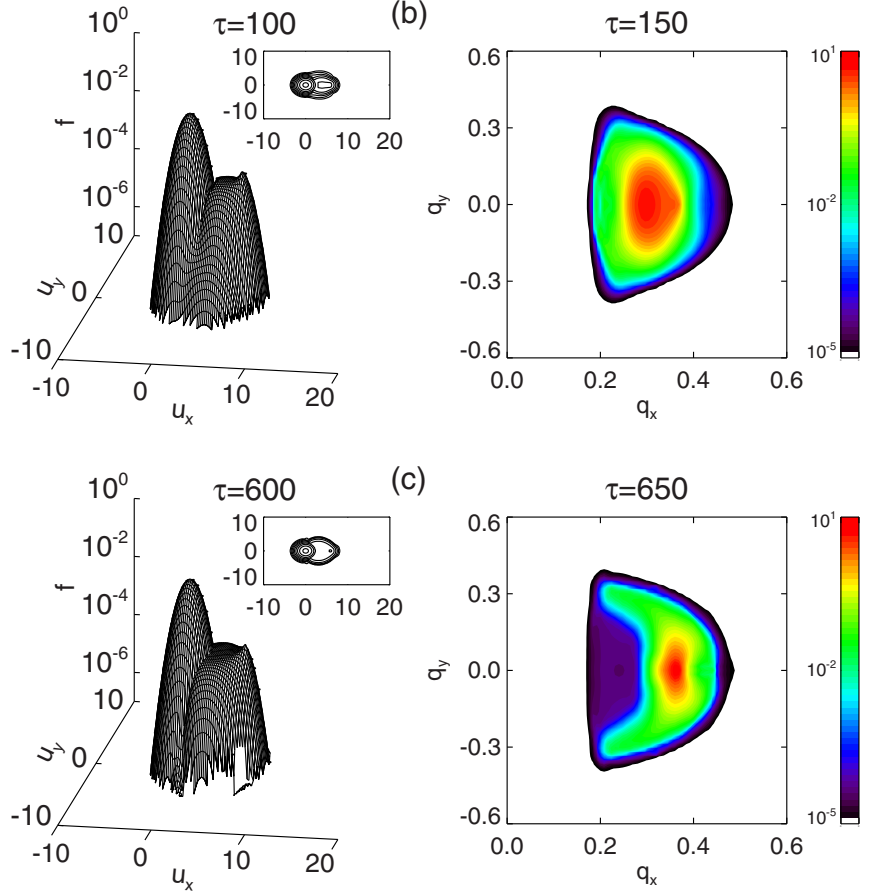

FIG. 4. (Color online) Electron distribution function, $f(\mathbf{u}, \tau)$, in $2 \mathrm{D}$ velocity space (left) and Langmuir wave intensity, $I(\mathbf{q}, \tau)$, in $2 \mathrm{D}$ wave vector space (right) obtained from the numerical solution of the 2D quasilinear equation.

D-shaped wave spectrum could be reproduced indicates that the formation of this spectrum shape is attributed to the linear wave-particle interaction. Of course, the waves with negative $q_{x}$ cannot be generated with linear process alone. The quasi-isotropic ring spectrum discussed by Ziebell et $a l^{22,23}$ must therefore rely on nonlinear processes. Nevertheless, it is important to note that the primary feature, namely, the D-shaped, or equivalently, an arc-shaped spectrum, can be largely accounted for on the basis of quasilinear theory.

There are some discrepancies between the theory and simulation. We find that the perpendicular diffusion of the beam is less prominent than in the simulation. The shift of the wave spectrum to higher $q_{x}$ and the broadening is smaller in the theoretical computation. We also find that the late time $(\tau=650)$ wave spectrum deviates from the intermediate $(\tau$ =150) D-shape spectrum. Finally, long-wavelength condensate mode is not excited. Although there is a feature which theory cannot explain, it is remarkable that the simple quasilinear theory can largely account for most of the important features in the PIC simulation.

Plotted in Fig. 5 are parallel and perpendicular electron 

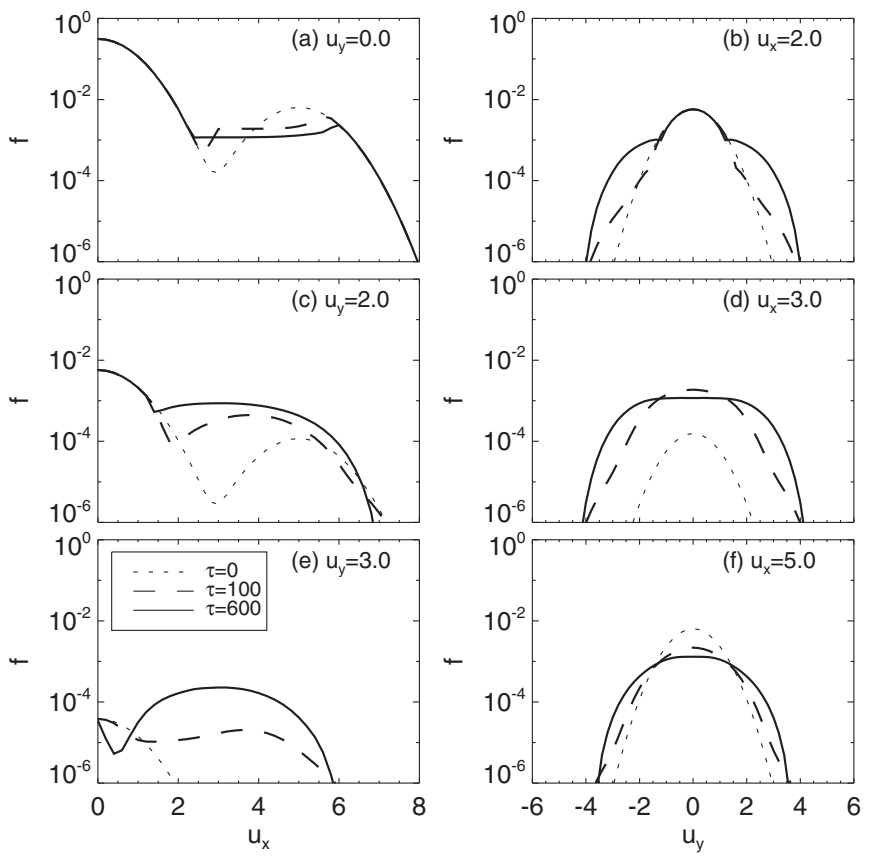

FIG. 5. Parallel and perpendicular electron distribution function as in Fig. 2. The left-hand panels are parallel cross sections with fixed (a) $u_{y}=0.0$, (c) $u_{y}=2.0$, and (e) $u_{y}=3.0$, and the right-hand panels correspond to perpendicular cross sections with fixed (b) $u_{x}=2.0$, (d) $u_{x}=3.0$, and (f) $u_{x}=5.0$. The distributions at three different times, $\tau=0,100$, and 600 are superposed.

distributions. The format is the same as Fig. 2. As one can see, major features discussed in Fig. 2 are reproduced. Of course, there are minor discrepancies, but the differences are mostly quantitative.

Parallel (left-hand panels) and perpendicular (right-hand panels) cross sections of the theoretical 2D wave spectrum are shown in Fig. 6 in the same format as in Fig. 3. For the
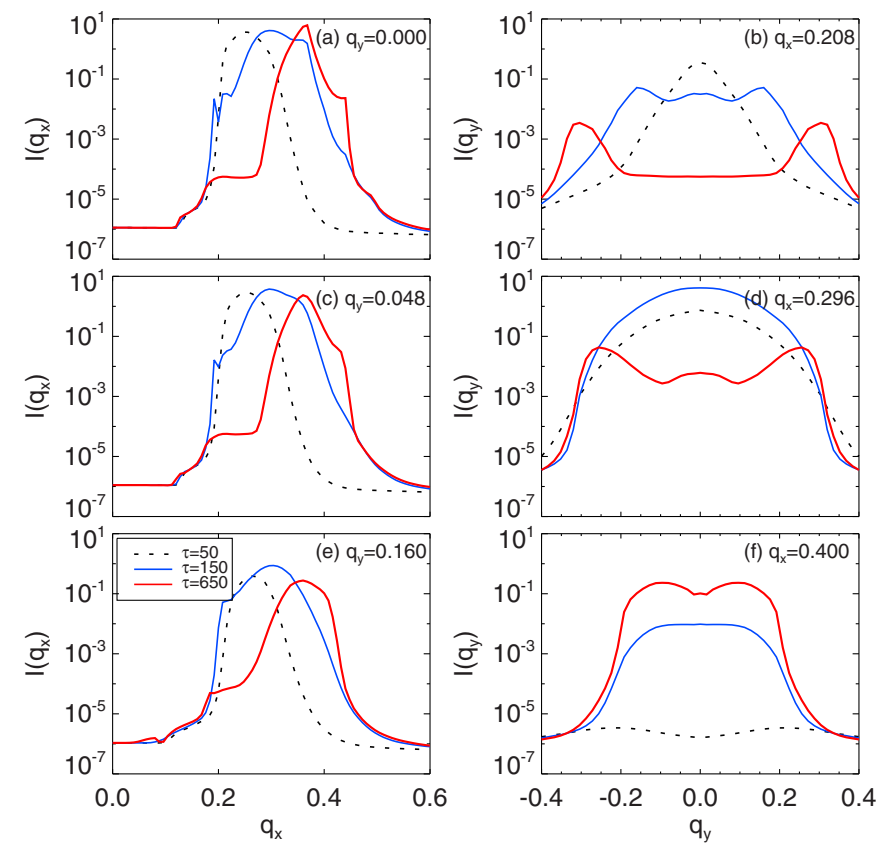

FIG. 6. (Color online) Parallel (left-hand panels) and perpendicular (righthand panels) cross sections of Langmuir wave intensity in the same format as Fig. 3.

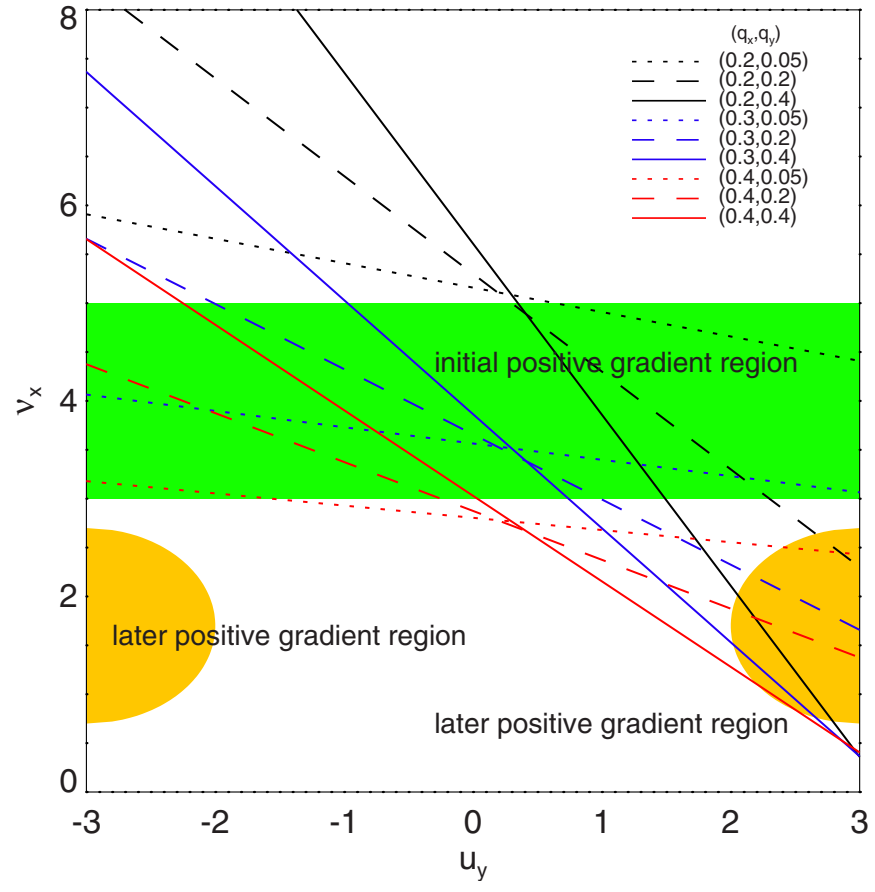

FIG. 7. (Color online) The parallel resonant velocity, $\nu_{x}$ as a function of the perpendicular velocity, $u_{y}$, for various wave numbers. The linear growth/ damping rate $\gamma_{\mathbf{q}}$ is determined from integration of $\left(\partial f / \partial u_{x}\right)_{u_{x}=\nu_{x}}$, where $\nu_{x}$ is defined by the resonance condition, $w_{\mathbf{q}}^{L}-\mathbf{q} \cdot \mathbf{u}=0$. Regions with initial positive gradient and those that develop later in time are indicated.

parallel cross section we chose (a) $q_{y}=0$, (c) $q_{y}=0.048$, and (e) $q_{y}=0.160$, while for the perpendicular cross section, we fixed (b) $q_{x}=0.208$, (d) $q_{x}=0.296$, and (f) $q_{x}=0.400$. Waves with $0.2<q_{x}<0.3$ and $\left|q_{y}\right|<0.3$ initially satisfy the resonant condition, $\delta\left(w_{\mathbf{q}}^{L}-\mathbf{q} \cdot \mathbf{u}\right)$, so, they start to grow and reach the maximum level around $\tau=150$. The saturated wave level $[I(\mathbf{q}) \approx 1]$ quantitatively agree with the simulation results. For later times, $\tau>200$, the waves generally decrease in intensity and shift to shorter parallel wavelength regime. This behavior is qualitatively consistent with the simulation. Since quasilinear theory excludes nonlinear effects, the longwavelength condensate mode is absent. Overall, the perpendicular cross sections of the wave intensity shown on the right-hand panels are also qualitatively consistent with the simulation.

\section{DISCUSSION}

In previous sections, we associated the dynamical evolution of the wave spectrum with the population inversion in the distribution function that develops late in time. In order to verify that such an association is indeed justified, we show in Fig. 7, the parallel velocities $\nu_{x}$ for various wave numbers. The growth/damping rate $\gamma_{\mathbf{q}}$ is computed by integrating $\left(\partial f / \partial u_{x}\right)_{u_{x}=\nu_{x}}$ along the paths defined by the resonance condition $w_{\mathbf{q}}^{L^{x}}-\mathbf{q} \cdot \mathbf{u}=0$ (they are shown as straight lines in Fig. 7). We also show regions with initial positive gradient and those that develop later in time. A significant portion of the integral path for a given wave number $\left(q_{x}, q_{y}\right)$ must overlap with the regions with positive gradient in order for $\gamma_{\mathbf{q}}$ to be 
positive. Note that the perpendicular derivative, $\left(\partial f / \partial u_{y}\right)_{u_{y}=\nu_{y}}$, always have negative value, so it only contributes to damping.

Figure 7 provides a graphic justification for the association between the change in the wave spectra and the dynamical evolution of the particle distribution function. For instance, the growth/damping rate for $\left(q_{x}, q_{y}\right)=(0.3,0.05)$ is initially positive since the integral path passes through the initial positive gradient region. At the later times, however, when the beam evolves into a plateau, the initial positive gradient disappears and the growth at $\left(q_{x}, q_{y}\right)=(0.3,0.05)$ turns to damping. In contrast, the wave number $\left(q_{x}, q_{y}\right)$ $=(0.4,0.05)$ corresponds to an initially damped mode. However, it changes to a growing mode later in time as a result of the late-time development of the parallel positive gradients with finite perpendicular velocities.

\section{CONCLUSIONS}

In the present paper we performed 2D PIC simulation for the beam-plasma instability, comparing with the $2 \mathrm{D}$ quasilinear theory. In the literature, the beam-plasma instability is usually studied in 1D, although some investigated higherdimensional effects. Among these are 2D and 3D analysis of quasilinear theory, ${ }^{17-19}$ 2D weak turbulence theory, ${ }^{22,23}$ and 2D PIC simulations. ${ }^{20,21}$ However, theoretical works and simulations were carried out separately and no efforts were made to compare the two approaches side by side. In this paper, we have carried out a detailed comparative study of the 2D simulation and 2D quasilinear theory in order fully assess the validity of the quasilinear theoretical approach, as well as the limitation thereof.

We found that the quasilinear theory can account for most relevant features in the PIC simulation result. For instance, the particle diffusion time scale, the maximum level of the excited waves, development of new positive gradients in the electron distribution at the later times, and change in the spectrum shape from an elliptical to D-shaped profile, all agree in a quantitative sense. However, the condensation phenomenon was not be able to be reproduced in the theory. It is noteworthy that the $2 \mathrm{D}$ weak turbulence theory performed by Ziebell et al. ${ }^{22,23}$ report that the $2 \mathrm{D}$ effects seem to inhibit the condensation, but the present 2D PIC code simulation shows that the Langmuir condensate is formed nevertheless. References 22 and 23 also report that over long time scale (on the order of several thousand plasma periods), the eventual Langmuir wave spectrum should be that of a quasi-isotropic ring spectrum in 2D wave number space. Our simulation was not carried out long enough to verify such a prediction, whose proof is left out for future study.

\section{ACKNOWLEDGMENTS}

The work was supported by Grant Nos. BK21, NRF2010-0023354, 2010-0020053, and 2010-0001840 from the
National Research Foundation of Korea. P.H.Y. acknowledges WCU Grant No. R31-10016 from the Korean Ministry of Education, Science and Technology to KHU, AFOSR Contract No. FA9550-07-0053 to the Massachusetts Technological Laboratory, Inc., and NSF Grant No. AGS0940985 to the University of Maryland.

${ }^{1}$ A. A. Vedenov, E. P. Velikhov, and R. Z. Sagdeev, Nucl. Fusion 2, 465 (1962).

${ }^{2}$ W. E. Drummond and D. Pines, Ann. Phys. (N.Y.) 28, 478 (1964).

${ }^{3}$ A. A. Vedenov and D. D. Ryutov, Reviews of Plasma Physics (Consultants Bureau, New York, 1975), Vol. 6.

${ }^{4}$ T. H. Stix, Waves in Plasmas (AIP, New York, 1992), p. 445.

${ }^{5}$ N. A. Krall and A. W. Trivelpiece, Principles of Plasma Physics (McGraw-Hill, New York, 1973), p. 512.

${ }^{6}$ B. B. Kadomtsev and V. I. Petviashvili, Sov. Phys. JETP 16, 1578 (1963).

${ }^{7}$ R. Z. Sagdeev and A. A. Galeev, Nonlinear Plasma Theory (Benjamin, New York, 1969), p. 89.

${ }^{8}$ D. B. Melrose, Plasma Astrophysics (Gordon and Breach, New York, 1980), p. 124.

${ }^{9}$ V. N. Tsytovich, Lectures on Nonlinear Plasma Kinetics (Springer-Verlag, New York, 1995), p. 107.

${ }^{10}$ P. H. Yoon, Phys. Plasmas 7, 4858 (2000).

${ }^{11}$ W. E. Drummond, J. H. Malmberg, and T. M. O'Neil, Phys. Fluids 13, 2422 (1970).

${ }^{12}$ T. M. O'Neil, J. H. Winfrey, and J. H. Malmberg, Phys. Fluids 14, 1204 (1971).

${ }^{13}$ T. M. O'Neil and J. H. Winfrey, Phys. Fluids 15, 1514 (1972).

${ }^{14}$ B. Li, A. J. Willes, P. A. Robinson, and I. H. Cairns, Phys. Plasmas 12, 012103 (2005).

${ }^{15}$ A. J. Willes, P. A. Robinson, and D. B. Melrose, Phys. Plasmas 3, 149 (1996).

${ }^{16}$ P. A. Robinson and D. L. Newman, Phys. Fluids B 1, 2319 (1989).

${ }^{17}$ V. L. Sizonenko and K. N. Stepanov, Zh. Eksp. Teor. Fiz. 49, 1197 (1965); Sov. Phys. JETP 22, 832 (1966).

${ }^{18}$ I. B. Bernstein and F. Engelmann, Phys. Fluids 9, 937 (1966).

${ }^{19}$ K. Appert, T. M. Tran, and J. Vaclavik, Phys. Rev. Lett. 37, 502 (1976).

${ }^{20}$ C. T. Dum and K.-I. Nishikawa, Phys. Plasmas 1, 1821 (1994).

${ }^{21}$ Y. Kasaba, H. Matsumoto, and Y. Omura, J. Geophys. Res. 106, 18693, doi:10.1029/2000JA000329 (2001).

${ }^{22}$ L. F. Ziebell, R. Gaelzer, and P. H. Yoon, Phys. Plasmas 15, 032303 (2008).

${ }^{23}$ L. F. Ziebell, R. Gaelzer, J. Pavan, and P. H. Yoon, Plasma Phys. Controlled Fusion 50, 085011 (2008).

${ }^{24}$ J. Pavan, L. F. Ziebell, R. Gaelzer, and P. H. Yoon, J. Geophys. Res. 114, A01106, doi:10.1029/2008JA013557 (2009).

${ }^{25}$ J. Pavan, L. F. Ziebell, P. H. Yoon, and R. Gaelzer, Plasma Phys. Controlled Fusion 51, 095011 (2009).

${ }^{26}$ J. Pavan, L. F. Ziebell, P. H. Yoon, and R. Gaelzer, J. Geophys. Res. 115, A01103, doi:10.1029/2009JA014447 (2010).

${ }^{27}$ J. Pavan, L. F. Ziebell, P. H. Yoon, and R. Gaelzer, J. Geophys. Res. 115, A02310, doi:10.1029/2009JA014448 (2010).

${ }^{28}$ P. H. Yoon, Phys. Plasmas 12, 042306 (2005).

${ }^{29}$ P. H. Yoon, T. Rhee, and C.-M. Ryu, Phys. Plasmas 12, 062310 (2005).

${ }^{30}$ H. Matsumoto and Y. Omura, Computer Space Plasma Physics: Simulation Techniques and Software (Terra Sci., Tokyo, 1993), p. 21.

${ }^{31}$ L. F. Ziebell, R. Gaelzer, and P. H. Yoon, Phys. Plasmas 8, 3982 (2001).

${ }^{32}$ P. H. Yoon, T. Rhee, and C.-M. Ryu, J. Geophys. Res. 111, A09106, doi:10.1029/2006JA011681 (2006).

${ }^{33}$ T. Rhee, C.-M. Ryu, and P. H. Yoon, J. Geophys. Res. 111, A09107, doi:10.1029/2006JA011682 (2006).

${ }^{34}$ W. H. Press, B. P. Flannery, S. A. Teukolsky, and W. T. Vetterling, $\mathrm{Nu}$ merical Recipes (Cambridge University Press, New York, 1986).

${ }^{35}$ P. H. Yoon, S. Yi, and C.-M. Ryu, Phys. Plasmas 12, 052305 (2005).

${ }^{36}$ C.-M. Ryu, T. Rhee, T. Umeda, P. H. Yoon, and Y. Omura, Phys. Plasmas 14, 100701 (2007). 